\title{
Corrective definition of traditional IF
}

Xue-Li Liu, Shuang-Shuang Gai, Jing Zhou

\section{Source}

Xue-Li Liu, Shuang-Shuang Gai, Jing Zhou. (2016). Journal Impact Factor: Do the

Numerator and Denominator Need Correction?. PLOS ONE, vol. 11 (3), e0151414.

$I F_{\text {Totall Total }}=\frac{C_{t-1}+C_{t-2}}{N_{t-1}+N_{t-2}}$

$C_{t-1}$ and $C_{t-2}$ are citations given in year t to all source items published in years (t-1) and (t2 ), respectively, $N_{t-1}$ and $N_{t-2}$ are the numbers of all source items published in years (t-1) and (t-2), respectively. 